\title{
9. SHARING THE RADIO SPECTRUM
}

\section{R. J. Cohen (NRAL Jodrell Bank)}

The radio spectrum is a common resource like air or water which can be used in many ways and for many purposes. Similarly it must be shared equitably. International use of radio is regulated by the International Telecommunications Union (ITU). The scientific use of radio for passive measurements such as radio astronomy or remote sensing (passive) was introduced into the ITU regulations at a late stage, and does not fit naturally into the framework. The needs of radio astronomy are inherently different from those of most other radio users.

The power levels reaching Earth from cosmic sources are generally very low and they are beyond the astronomer's control. Large antennas and sensitive receivers are needed to detect and measure them. This makes radio astronomy particularly vulnerable to interference from man-made emissions. The cosmic emissions are often of unknown character, such is the nature of research, and this can make it difficult to distinguish the wanted signal from interference. Radio astronomy needs quiet frequency bands in which to conduct measurements of the highest sensitivity. This is best achieved by world-wide allocations of passive frequency bands. Yet many other frequency bands are also of scientific interest. Only $2 \%$ of the radio spectrum below $50 \mathrm{GHz}$ is allocated to radio astronomy, and only 30 allocations are passive.

As the worldwide use of radio expands, radio astronomy is increasingly going to be confined to the officially allocated bands. Concern is now mounting at the state of those bands. Real transmitters cannot confine their emissions to sharply-defined frequency bands like those allocated by the ITU. Inevitably there are unwanted emissions which spill into other frequency bands, creating a type of radio pollution. The ITU has been slow to address this growing problem of unwanted emissions. Emissions from satellites are a particular concern for radio astronomy. Just one satellite can affect radio observatories all around the world, however isolated geographically and however well-shielded from terrestrial transmitters. The ITU has no regulations on the unwanted emissions from satellite transmitters.

Examples of interference to radio astronomy from satellites are growing steadily. In the 1980s the global satellite navigation systems GPS and GLONASS became well known to radio astronomers throngh their unwanted emissions into the frequency bands around $1.6 \mathrm{GHz}$ used to observe spectral lines of $\mathrm{OH}$. The solution to such problems takes many years to negotiate and to implement, once the system is already flying. This year the Iridium communications satellite system looks set to cause interference in the very same OH band, despite the experiences of GLONASS and despite efforts to avoid a similar problem with Iridium. At higher frequencies we have the Astra satellite over Europe interfering at $10.7 \mathrm{GHz}$ with observations in a passive band.

My great concern is for the future. Altogether $80 \%$ of radio astronomy bands with a worldwide primary allocation are immediately adjacent to bands allocated for satellite downlinks! GLONASS and Astra may be only the beginning. The low Earth orbitting satellites (LEOs) of the future will fly in large constellations like Iridium, so that many will be visible simultaneously. The challenge will need to be addressed at many levels, from technical to regulatory. We are not opposed to satellite systems, but we do not want their downlinks right next to our spectral lines. We cannot stop the telecommunications revolution, but we must find ways to reduce its impact on radio astronomy.

\section{KEEPING THE RADIO WINDOWS OPEN}

\section{W. A. Baan (Arecibo Observatory, IUCAF Secretariat Barrio Esperanza)}

Passive scientific use of the radio spectrum has become more difficult due to harmful interference from active users in other spectral bands. The time has come for national administrations and spectrum managers to re-consider the protection afforded the non-profit passive scrvices from market-oriented commercial users. The following urgent issues need to be addressed in order to assure adequate protection for the passive users operating in the Radio Astronomy and Earth Exploration services:

1. The Radio Regulations of the International Telecommunication Union - The Radio Regulations (RR) afford protection of all spectrum users on an equal basis and do not allow preferential treatment of one particular service. However, the passive Radio Astronomy and Earth Exploration users of the spectrum only observe natural emissions that are significantly weaker than the vast majority of man-made signals. Therefore, this "equality for all" rule from the RR camnot 